\title{
Consenso | Protocolo Brasileiro para Infecções Sexualmente Transmissíveis 2020: violência sexual
}

doi: 10.1590/S1679-4974202100018.esp1

\author{
Brazilian Protocol for Sexually Transmitted Infections 2020: sexual violence \\ Protocolo Brasileño para Infecciones de Transmisión Sexual 2020: violencia sexual
}

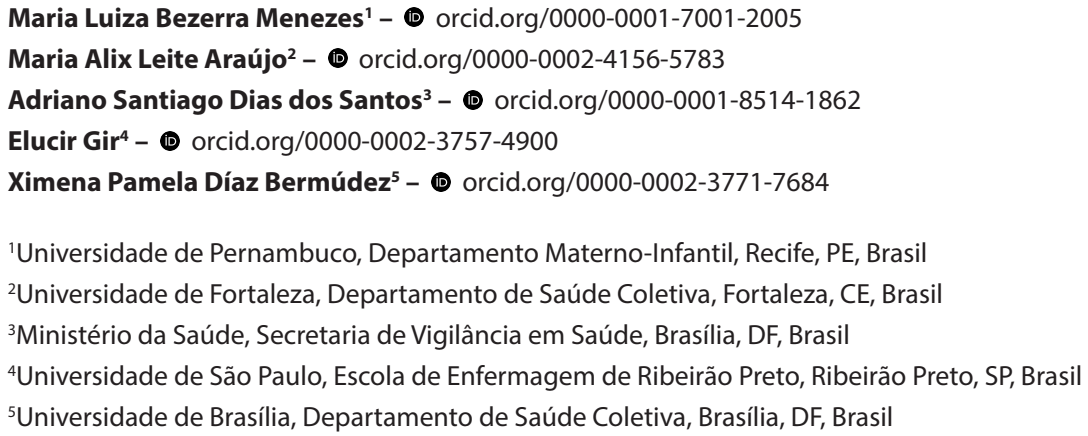

\section{Resumo}

Este artigo aborda a violência sexual, tema que compõe o Protocolo Clínico e Diretrizes Terapêuticas para Atenção Integral às Pessoas com Infecções Sexualmente Transmissíveis, publicado pelo Ministério da Saúde do Brasil. São apresentadas orientações aos gestores para o manejo programático e operacional, com foco na rede de atendimento às pessoas em situações de violência sexual, recomendações aos profissionais de saúde acerca de medidas profiláticas de gravidez e de infecções sexualmente transmissíveis virais e não virais, além das estratégias para as ações de vigilância. A violência sexual constitui um problema amplo, extrapola o campo de saúde e envolve desafios conceituais e programáticos tanto para os profissionais de saúde, na linha de frente na atenção às pessoas afetadas, quanto para a sociedade, em termos de possíveis formas de prevenção.

Palavras-chave: Delitos Sexuais; Violência por Parceiro Íntimo; Doenças Sexualmente Transmissíveis; Protocolos Clínicos.

Endereço para correspondência:

Adriano Santiago Dias dos Santos - Ministério da Saúde, Secretaria de Vigilância em Saúde, SRTVN, Quadra 701, Via W 5 Norte, Lote D, Edifício PO 700, Brasília, DF, Brasil. CEP 70719-040

E-mail: adriano.santos@aids.gov.br 


\section{Apresentação}

0 artigo aborda a violência sexual, tema que compõe o Protocolo Clínico e Diretrizes Terapêuticas (PCDT) para Atenção Integral às Pessoas com Infecções Sexualmente Transmissíveis (IST), além do PCDT para Profilaxia Pós-Exposição de Risco à Infecção pelo HIV, IST e Hepatites Virais e do PCDT para Manejo da Infecção pelo HIV em Crianças e Adolescentes, publicados pelo Ministério da Saúde do Brasil. Para a elaboração dos protocolos, foram realizadas a seleção e a análise das evidências disponíveis na literatura e sua discussão, em um painel de especialistas. 0 PCDT para Atenção Integral às Pessoas com IST foi aprovado pela Comissão Nacional de Incorporação de Tecnologias no Sistema Único de Saúde (Conitec) ${ }^{1}$, sendo atualizado pelo grupo de especialistas em IST em 2020.

\section{A violência sexual constitui uma das principais formas de violação dos direitos humanos, atingindo o direito à vida, à saúde eà integridade física.}

\section{Introdução}

Conceitua-se violência sexual como "qualquer ato sexual, tentativa de obter um ato sexual, comentários ou investidas sexuais indesejadas, ou atos de tráfico sexual ou de outra forma direcionados contra a sexualidade de uma pessoa, por meio de coerção, por qualquer pessoa, independentemente de seu relacionamento com a vítima, em qualquer ambiente, incluindo, mas não se limitando à casa e ao trabalho". ${ }^{2}$

A violência sexual é problema estrutural de diversas sociedades contemporâneas, muitas vezes negligenciado, podendo afetar pessoas de ambos os sexos e todas as faixas etárias, com graves consequências físicas e traumas emocionais tanto para as vítimas como para seus familiares. ${ }^{3}$ Trata-se de um evento multidimensional, presente em todas as classes sociais, raças e etnias, relações de gênero e orientação sexual. Ela constitui uma das principais formas de violação dos direitos humanos, atingindo o direito à vida, à saúde e à integridade física. ${ }^{4-5}$

0 enfrentamento da violência sexual requer a participação de órgãos das áreas da saúde, educação, trabalho, segurança pública, justiça, e de direitos humanos, e exige políticas públicas intersetoriais e ações integradas do Estado e da sociedade geral. Destaca-se a importância do respeito à diversidade e às identidades de gênero, garantindo-se 0 acesso aos direitos em todas as áreas e privilegiando-se a saúde das pessoas afetadas. ${ }^{6-7}$

Um dos tipos graves, e mais comuns, de violência sexual é o estupro, definido como "penetração física forçada ou coagida, mesmo que leve, na vulva ou ânus, usando o pênis, outras partes do corpo ou um objeto". 0 crime de estupro é definido no Código Penal brasileiro como o "ato de constranger alguém, mediante violência ou grave ameaça, a ter conjunção carnal ou a praticar ou permitir que com ele se pratique outro ato libidinoso".

0 tema da violência sexual tem alcançado notoriedade. Todavia, faz-se necessário propor agendas de pesquisa que contribuam com a produção de evidências científicas nesse campo. ${ }^{6,9,10}$ Registros apontam que o sexo feminino é o mais afetado, representando mais de $80 \%$ das vítimas de violência sexual. ${ }^{6}$ No Brasil, entre os anos de 2011 e 2018, foram notificados 1.282.045 casos de violência contra mulheres.?

Embora a literatura científica reconheça que a violência sexual afeta majoritariamente as mulheres, é importante também dar luz aos eventos de violência sexual contra vítimas do sexo masculino. Estudo que analisou dados de estupro nos anos de 2017 e 2018 identificou que a faixa etária mais afetada no sexo masculino foi a de crianças de 5 a 9 anos, representando cerca de um quarto dos casos, e que 0 auge da vitimização ocorreu em meninos com 7 anos de idade. ${ }^{6}$ Não se deve considerar apenas a notificação desses eventos e suas consequências físicas e psicossociais, sugerindo-se, também, o aprofundamento de estudos que analisem 0 vácuo empírico dessa realidade. ${ }^{11}$ Observa-se que, embora aspectos relativos à própria constituição das masculinidades predisponham os homens ao não reconhecimento da experiência desse tipo de evento, situações de conflitos armados, deslocamentos e migrações apresentam alta incidência de violência sexual afetando homens. ${ }^{12-14}$

Pesquisa e análise sobre a ocorrência de violência sexual no ano de 2019 no Brasil mostrou que somente $7,5 \%$ desses crimes são notificados às autoridades policiais, ${ }^{15}$ quanto ao estupro, mais de 60\% deles são cometidos contra vulneráveis, ou seja, pessoas com idade inferior a 14 anos, consideradas 
juridicamente incapazes para consentir relação sexual. Na mesma categoria encontram-se as pessoas incapazes de oferecer resistência, independentemente de sua idade, como alguém que esteja sob efeito de drogas, enfermo ou apresente alguma deficiência, como determina a Lei ${ }^{0} 12.015$, de 7 de agosto de 2009. 0 mesmo estudo citado evidencia a predominância de agressores do sexo masculino em mais de $80 \%$ dos casos. ${ }^{15}$

É necessário considerar que a violência sexual também afeta pessoas transexuais, um grupo social de alta vulnerabilidade para violência e para IST. Revisão sistemática, baseada em 74 estudos junto a essa população, desenvolvidos no Brasil e em outros países, revelou que o estigma e o preconceito estão relacionados à identidade de gênero e à orientação sexual, expressos na alta prevalência de eventos de violência física e sexual contra esse grupo social. ${ }^{16}$

Uma das consequências da violência sexual é a possibilidade de transmissão de IST, ${ }^{17}$ o que provoca medo e ansiedade nas vítimas, especialmente quando está relacionada ao vírus da imunodeficiência humana (buman immunodeficiency virus, HIV). Por esse motivo, deve-se ofertar assistência imediata à vítima por meio de atendimento clínico-laboratorial, profilaxia pós-exposição (PEP) de risco para infecção pelo HIV, hepatites virais e IST não virais (gonorreia, sífilis, infecção por clamídia, tricomoníase e cancroide), atendimento psicológico e social, prevenção de gravidez indesejada, além das orientações adequadas sobre os procedimentos médicos e direitos legais. ${ }^{18}$

Estudo transversal que estimou a ocorrência de gravidez e IST decorrente de violência sexual no estado de Santa Catarina, entre os anos de 2008 e 2013, identificou que mais de $7 \%$ das vítimas engravidaram e mais de $3 \%$ foram acometidas por uma IST. ${ }^{17} 0$ risco dessas infecções depende do tipo de exposição (vaginal, anal ou oral), número de agressores, recorrência da exposição, ocorrência de traumatismos genitais, idade da vítima e sua susceptibilidade (condição himenal e presença prévia de outras IST). ${ }^{17}$

A violência sexual, quando praticada pela parceria íntima, envolve afetividade, tornando seu enfrentamento ainda mais desafiador. 0 ato está relacionado a fatores sociais, culturais e econômicos e, muitas vezes, sua ocorrência é favorecida por valores, ideologias e normas que a naturalizam. ${ }^{3,19}$ Intervêm nesses processos relações estruturais, iniquidades de gênero, fatores individuais e formas de compreensão da violência por parte da sociedade. ${ }^{20}$
Por essas razões, além do pleno conhecimento e implementação das diretrizes terapêuticas de parte dos profissionais de saúde, deve-se garantir 0 acesso das vítimas de violência sexual aos diferentes serviços de apoio ao processo de recuperação e cura do trauma, em uma perspectiva abrangente da saúde física, mental e sexual. ${ }^{21}$

\section{Profilaxia das infecções sexualmente transmissíveis em situações de violência sexual}

0 atendimento após a ocorrência de violência sexual é uma urgência, para a qual se deve garantir acesso e acolhimento, reconhecendo as especificidades das populações-chave e prioritárias. Esse atendimento deve ser realizado em local adequado, com garantia de privacidade e sem julgamentos morais. Uma avaliação inicial do paciente deve incluir um diálogo acerca do contexto relacionado à situação da violência e à pertinência da prescrição da profilaxia. ${ }^{22,23}$

Recomenda-se a iniciação imediata da profilaxia das IST não virais em todos os casos de violência sexual, sempre que possível. ${ }^{22}$ Em gestantes, a profilaxia pode ser administrada em qualquer idade gestacional. ${ }^{18}$ Eventualmente, o esquema profilático pode ser postergado em função de algumas condições que dificultem a adesão, como pessoas em situação de extrema fragilidade física ou emocional, ou pode ser evitado, em casos de intolerância gastrintestinal aos medicamentos. 0 esquema profilático para IST não virais em situações de violência sexual é apresentado na Figura 1.

A PEP para HIV, hepatites virais e outras IST é uma estratégia de prevenção ofertada pelo Sistema Único de Saúde (SUS) e consiste no uso de medicamentos para reduzir o risco de adquirir essas infecções. ${ }^{18,24}$ A PEP para HIV é uma tecnologia inserida no conjunto de estratégias da chamada Prevenção Combinada, cujo principal objetivo é ampliar as formas de intervenção para evitar novas infecções pelo HIV. ${ }^{18}$ A Prevenção Combinada conjuga diferentes ações e contempla a combinação das intervenções biomédica, comportamental e estrutural, aplicadas aos âmbitos individual e coletivo. ${ }^{25}$ A PEP para o HIV consiste no uso de antirretrovirais por um período de 28 dias e deve-se iniciar, no máximo, até 72 horas após a exposição. 0 esquema preferencial deve incluir combinações de três ou quatro antirretrovirais, devendo ser composto por dois 


\begin{tabular}{|c|c|c|c|}
\hline \multirow{2}{*}{$\begin{array}{l}\text { Infecção sexualmente } \\
\text { transmissível }\end{array}$} & \multirow[b]{2}{*}{ Medicamento } & \multicolumn{2}{|c|}{ Posologia } \\
\hline & & $\begin{array}{l}\text { Adultos e adolescentes com mais } \\
\text { de } 45 \mathrm{~kg} \text {, inclusive gestantes }\end{array}$ & $\begin{array}{l}\text { Crianças e adolescentes com } \\
\text { menos de } 45 \mathrm{~kg}\end{array}$ \\
\hline Sífilis & Benzilpenicilina benzatina & $\begin{array}{l}\text { 2,4 milhões de unidades internacionais } \\
\text { (UI), intramuscular (IM) em dose única } \\
\text { (dividida em duas injeções) }\end{array}$ & $\begin{array}{l}50.000 \text { UI/kg, IM, dose única (dose } \\
\text { máxima total: } 2,4 \text { milhões UI) }\end{array}$ \\
\hline Gonorreia & Ceftriaxona + azitromicina & $\begin{array}{l}\text { Ceftriaxona 500mg, } 1 \text { ampola, IM, } \\
\text { dose única }\end{array}$ & $+/+++$ \\
\hline Infecção por clamídia & Azitromicina & $\begin{array}{l}\text { 500mg, } 2 \text { comprimidos, V0, dose única } \\
\text { (dose total: } 1 \mathrm{~g} \text { ) }\end{array}$ & $\begin{array}{l}20 \mathrm{mg} / \mathrm{kg} \text { de peso, } V 0 \text {, dose única (dose } \\
\text { máxima total: } 1 \mathrm{~g} \text { ) }\end{array}$ \\
\hline Tricomoníase & Metronidazol ${ }^{a, b}$ & $\begin{array}{l}\text { 500mg, } 4 \text { comprimidos V0, dose única } \\
\text { (dose total: } 2 \mathrm{~g} \text { ) }\end{array}$ & $\begin{array}{l}15 \mathrm{mg} / \mathrm{kg} / \text { dia, divididos em } 3 \text { doses/ } \\
\text { dia, por } 7 \text { dias (dose diária máxima: } 2 \mathrm{~g} \text { ) }\end{array}$ \\
\hline
\end{tabular}

Fonte: adaptado do Protocolo Clínico e Diretrizes Terapêuticas para Atenção Integral às Pessoas com Infecções Sexualmente Transmissíveis. ${ }^{20}$

Notas: a) A administração profilática do metronidazol ou suas alternativas pode ser postergada ou evitada em casos de intolerância gastrintestinal ao medicamento conhecida; e deve ser postergada nos casos em que houver prescrição de contracepção de emergência e de profilaxia pós-exposição; b) 0 metronidazol não poderá ser utilizado no primeiro trimestre de gestação.

\section{Figura 1 - Esquema profilático para infecções sexualmente transmissiveis não virais em situações de violência sexual}

inibidores de transcriptase reversa nucleosídeos, preferencialmente coformulados, associados a outra classe, geralmente inibidores de integrase, preferencialmente dolutegravir, ou inibidores de protease com ritonavir, como adjuvante farmacológico. ${ }^{18,26,27} \mathrm{~A}$ apresentação e as posologias de antirretrovirais preferenciais, recomendados no Brasil para PEP, encontram-se na Figura $2 .^{18}$

Para as gestantes, o esquema preferencial de terapia antirretroviral deve ser tenofovir e lamivudina, composto com atazanavir/ritonavir nas doses de atazanavir $300 \mathrm{mg}$ (um comprimido) e ritonavir $100 \mathrm{mg}$ (um comprimido), via oral, ambos uma vez ao dia, ou raltegravir na dose de $400 \mathrm{mg}$ a partir da $14^{\mathrm{a}}$ semana de gestação (um comprimido, via oral, duas vezes ao dia)..$^{18}$

Mulheres lactantes devem ser orientadas sobre os potenciais riscos de transmissão do HIV pelo leite materno ${ }^{28,29}$ e, nos casos em que sofreram violência sexual, orientadas para a interrupção temporária da amamentação. Durante o período de janela imunológica, recomenda-se a extração e descarte do leite; uma vez realizado um exame de controle para o HIV na $12^{a}$ semana após início da PEP e sendo seu resultado não reagente, autoriza-se a reintrodução do aleitamento materno. ${ }^{18}$

Na prática clínica, o diagnóstico de abuso sexual em bebês, crianças e adolescentes é difícil e depende, também, da atenção e sensibilidade do profissional de saúde. ${ }^{30} 0$ s comportamentos possivelmente indicativos de que uma criança foi abusada sexualmente incluem perpetração sexual ou brincadeiras inadequadas a seu desenvolvimento, como tocar repetidamente nos órgãos genitais de um adulto ou pedir a um adulto que toque em seus genitais. ${ }^{30}$

Em que pesem essas manifestações, evidências indicam que a violência sexual em crianças permanece invisível aos serviços de saúde. ${ }^{31}$ Crianças apresentam maior vulnerabilidade às IST devido, entre outros fatores, à imaturidade anatômica e fisiológica da mucosa gênito-anal. 0 diagnóstico de uma IST em uma criança pode ser o primeiro sinal de abuso sexual e, portanto, deve ser investigado. ${ }^{32,33}$ A maioria das queixas são inespecíficas; porém, sangramento ou trauma retal ou genital, e IST não adquiridas no período perinatal, por transmissão vertical, devem chamar a atenção do profissional de saúde. ${ }^{33}$

Os esquemas e posologias da PEP para o HIV nessa faixa etária, especialmente nas crianças com menos de 12 anos, devem ser ajustados. ${ }^{15}$ Para crianças com mais de 12 anos, considerando-se a segurança e facilidade de prescrição, recomenda-se tenofovir e lamivudina associados ao dolutegravir. ${ }^{18,34}$

Ressalta-se que os adolescentes têm direito à PEP mesmo sem a presença dos pais ou responsáveis legais. Nesses casos, conforme previsto no Estatuto da Criança e do Adolescente - Lei n ${ }^{\circ} 8.069$, de 13 de julho de 1990 -, deve-se avaliar a capacidade de discernimento do adolescente, salvo nas situações de violência. ${ }^{35}$

Se a pessoa em situação de violência referir que não foi vacinada ou está com o esquema vacinal incompleto contra hepatite B, deve-se administrar a primeira dose da vacina ou completar o esquema vacinal. 


\begin{tabular}{|l|l|l|}
\hline Medicamento & Apresentação & Posologia \\
\hline \multirow{2}{*}{ Tenofovira + Lamivudina } & $\begin{array}{l}\text { Comprimido coformulado (tenofovir 300mg + } \\
\text { lamivudina 300mg }\end{array}$ & 1 comprimido via oral (V0) 1x/dia \\
\cline { 2 - 3 } & $\begin{array}{l}\text { Na indisponibilidade da apresentação coformulada: } \\
\text { comprimido tenofovir 300mg + comprimido } \\
\text { lamivudina 150mg }\end{array}$ & $\begin{array}{l}\text { Na indisponibilidade da apresentação coformulada: } \\
1 \text { comprimido V0 1x/dia + 2 comprimidos V0 1x/dia }\end{array}$ \\
\hline Dolutegravir & Comprimido dolutegravir 50mg & 1 comprimido V0 1x/dia \\
\hline
\end{tabular}

Fonte: adaptado do Protocolo Clínico e Diretrizes Terapêuticas para Profilaxia Pós-Exposição de Risco à Infecção pelo HIV, IST e Hepatites Virais. ${ }^{15}$

Nota: a) Contraindicado para pessoas com insuficiência renal aguda e na pré-concepção; em mulheres em idade fértil, descartar gravidez e orientar o uso regular de contraceptivo eficaz; não recomendado para pessoas em uso de fenitoína, fenobarbital, oxicarbamazepina, carbamazepina, dofetilida e pilsicainida.

\section{Figura 2 - Apresentação e posologias de antirretrovirais preferenciais para profilaxia pós-exposição}

Não se recomenda o uso rotineiro de imunoglobulina humana anti-hepatite $\mathrm{B}$, exceto se a vítima for suscetível, e se o responsável pela violência for reagente ao antígeno de superfície do vírus da hepatite B (HBsAg) ou pertencente a um grupo de risco, como, por exemplo, pessoas que usam drogas ilícitas. Quando indicada, a imunoglobulina humana anti-hepatite B deve ser aplicada o mais precocemente possível, até, no máximo, 14 dias após a exposição. ${ }^{18}$

\section{Prevenção da gravidez em situação de violência sexual}

Entre as etapas do atendimento à mulher vítima de violência sexual, constam as orientações acerca da prevenção da gravidez indesejada. Para aquelas que decidirem por essa profilaxia, deve-se prescrever e disponibilizar levonorgestrel na dose de um comprimido de $1,5 \mathrm{mg}$ via oral ou dois comprimidos de $0,75 \mathrm{mg}$, dose única (ou fracionada em duas tomadas a cada 12 horas), até cinco dias após a relação sexual. Esse método propicia vantagens sobre o método de Yuzpe (administração de contraceptivos hormonais combinados na dosagem de $200 \mathrm{mcg}$ de etinil estradiol e $1 \mathrm{mg}$ de levonorgestrel em dose única ou dividida em duas tomadas, com intervalo de 12 horas),$^{36}$ haja vista sua maior eficácia, pois apresenta falha em apenas $2 \%$ a 3\% dos casos, e segurança, pela menor ocorrência de interações medicamentosas e baixa ocorrência de efeitos colaterais. ${ }^{36,37}$

Quando, inevitavelmente, a gravidez decorre de violência sexual, o abortamento é permitido pelo Decreto-Lei $\mathrm{n}^{0} 2.848$, de 7 de dezembro de 1940, artigo 128, inciso II do Código Penal brasileiro e outras normas infralegais. 8,38

\section{Assistência e articulação da rede de atendimento em situação de violência sexual}

0 atendimento às pessoas em situação de violência sexual no SUS deve estar em conformidade com a norma técnica vigente do Ministério da Saúde. ${ }^{21}$ Ele deve se realizar conforme a Lei $\mathrm{n}^{0} 12.845$, de $1^{\circ}$ de agosto de $2013^{39}$ (dispõe sobre 0 atendimento obrigatório e integral pelo SUS), o Decreto $\mathrm{n}^{0} 7.958$, de 13 de março de $2013^{40}$ (estabelece diretrizes para 0 atendimento pelos profissionais da Segurança Pública e da rede de atendimento do SUS), e 0 art. $5^{\circ}$ da Portaria GM/MS n ${ }^{\circ}$ 485, de $1^{0}$ de abril de $2014^{41}$ (redefine o funcionamento do serviço de atenção às pessoas nessas situações).

0 serviço de saúde, em geral, pode representar um espaço privilegiado para identificação de uma pessoa que sofreu ou sofre violência sexual, desde que o profissional de saúde esteja sensível e atento a determinados sinais e sintomas apresentados durante 0 atendimento. Uma vez identificada a violência sexual, cumpre a esse profissional encaminhar a vítima para um serviço especializado na oferta de atendimento emergencial e integral. Nesse tipo de serviço, a pessoa é acolhida por uma equipe multidisciplinar, recebe medicamentos e as orientações necessárias.

Destaca-se a importância da escuta e registro qualificado do fato ocorrido, realização de exames clínicos, ginecológicos, laboratoriais, coleta de vestígios, contracepção de emergência, profilaxias para IST virais (HIV e hepatite B) e não virais, notificação epidemiológica compulsória em 24 horas (ficha de notificação da violência), acompanhamento social, psicológico e seguimento ambulatorial. ${ }^{22,42}$ 
A assistência à pessoa em situação de violência sexual demanda a estruturação das redes de atenção de forma intersetorial, articulada e comprometida, oferecendo acesso ao cuidado qualificado. 0s órgãos governamentais responsáveis pelas políticas de saúde e segurança devem identificar as organizações e serviços disponíveis no território, aptos a contribuir com essa assistência: Delegacia da Mulher, Delegacia da Criança e do Adolescente, Conselho Tutelar, Conselho dos Direitos da Criança e do Adolescente, Centro de Referência de Assistência Social, Instituto Médico Legal, Ministério Público, Casas Abrigo, grupos de mulheres, creches, entre outros. 0 fluxo e os problemas de acesso e de manejo dos casos, em cada nível dessa rede, precisam ser debatidos e planejados periodicamente. . $3,44^{4}$

Ainda que o município não disponha de uma rede de saúde totalmente estruturada, com disponibilidade de todos os serviços necessários para 0 atendimento integral de pessoas que sofrem violência sexual, é importante que ele conte com uma infraestrutura mínima e equipes capacitadas para 0 atendimento inicial, e que esteja articulado com outros municípios para garantir o seguimento em serviços especializados. ${ }^{7}$

A Figura 3 apresenta 0 detalhamento de algumas etapas essenciais para a materialização da rede de cuidados e de proteção social (intrassetorial ou

\begin{tabular}{|c|c|}
\hline Etapa & Estruturação da rede intrassetorial e intersetorial \\
\hline \multirow{3}{*}{$\begin{array}{l}\text { Diagnóstico da } \\
\text { situação }\end{array}$} & $\begin{array}{l}\text { Mapeamento dos serviços governamentais e não governamentais que atuam no cuidado integral, na promoção, defesa e } \\
\text { proteção dos direitos das pessoas no território, inclusive serviços de atendimento ao autor da agressão. }\end{array}$ \\
\hline & Identificação dos serviços de "porta de entrada" para atenção integral às pessoas em situação de violências. \\
\hline & $\begin{array}{l}\text { Protocolos e fluxos de atendimento; articulação em rede intrassetorial e intersetorial; tipo de atendimento prestado; } \\
\text { endereço, telefones, e-mail, horário de atendimento. }\end{array}$ \\
\hline \multirow{3}{*}{$\begin{array}{l}\text { Mobilização social e } \\
\text { direitos }\end{array}$} & $\begin{array}{l}\text { Pactuação com a gestão local sobre a rede de cuidado e de proteção social para a atenção integral às pessoas em situação de } \\
\text { violências. }\end{array}$ \\
\hline & Formalização de atos normativos. \\
\hline & $\begin{array}{l}\text { Alianças estratégicas com Conselhos Tutelares e de Direitos; Centro de Referência Especializado de Assistência Social (CREA); } \\
\text { Centro de Referência de Assistência Social (CRAS); associações comunitárias; meios de comunicação; Ministério Público, } \\
\text { Segurança Pública; Poderes Legislativo e Judiciário. }\end{array}$ \\
\hline \multirow{3}{*}{$\begin{array}{l}\text { Educação } \\
\text { permanente }\end{array}$} & $\begin{array}{l}\text { Sensibilização e capacitação de profissionais de saúde dos três níveis de atenção para acolhimento, atendimento, notificação } \\
\text { e seguimento dos casos. }\end{array}$ \\
\hline & $\begin{array}{l}\text { Articulação com os serviços de promoção, proteção e defesa dos direitos das pessoas em situação de violências para a atenção } \\
\text { integral à saúde na formação continuada. }\end{array}$ \\
\hline & $\begin{array}{l}\text { Articulação com as instituições de ensino e pesquisa para a inserção do tema da prevenção de violências e promoção da } \\
\text { cultura de paz nas disciplinas dos cursos de saúde, assistência social e educação. }\end{array}$ \\
\hline \multirow{6}{*}{$\begin{array}{l}\text { Planejamento e } \\
\text { gestão }\end{array}$} & $\begin{array}{l}\text { Criação de grupo de gestão colegiada da rede de cuidado e de proteção social das pessoas em situação de violências e suas } \\
\text { famílias, para articular, mobilizar, planejar, acompanhar e avaliar as ações da rede. }\end{array}$ \\
\hline & $\begin{array}{l}\text { Planejamento das ações e estratégias para atenção integral à saúde das pessoas em situação de violências, com base na } \\
\text { realidade local. }\end{array}$ \\
\hline & Elaboração/atualização de protocolos de acolhimento e atendimento humanizados. \\
\hline & $\begin{array}{l}\text { Adoção de estratégias de acompanhamento e apoio técnico e psicossocial às equipes de saúde que atendem pessoas } \\
\text { envolvidas em situação de violência; e estímulo à rede intersetorial, para que também siga essas estratégias. }\end{array}$ \\
\hline & $\begin{array}{l}\text { Implantação/implementação do sistema de vigilância de violências e acidentes 'Viva Contínuo' no Sistema de Informação de } \\
\text { Agravos de Notificação (Sinan). }\end{array}$ \\
\hline & $\begin{array}{l}\text { Comunicação e divulgação à sociedade da relação de serviços, com endereço completo e horários de atendimento, às pessoas } \\
\text { em situaça de violências (Guia de Serviços). }\end{array}$ \\
\hline
\end{tabular}

Fonte: Protocolo Clínico e Diretrizes Terapêuticas para Atenção Integral às Pessoas com Infecções Sexualmente Transmissíveis. ${ }^{20}$

\section{Figura 3 - Estrutura da rede de cuidados e de proteção social, intrassetorial e intersetorial, às pessoas em situação de violência sexual}


intersetorial), que não necessariamente seguem uma hierarquia e podem acontecer de forma concomitante. Os serviços de atenção às pessoas em situação de violência sexual devem estar registrados no Sistema do Cadastro Nacional de Estabelecimentos de Saúde (SCNES), com o código $165 .{ }^{44}$

As instituições de saúde devem informar a disponibilização de: (i) atenção integral às pessoas em situação de violência sexual (classificação 001 do serviço especializado 165), que pode ser organizada em hospitais gerais e maternidades, prontos-socorros, Unidades de Pronto Atendimento (UPA) e no conjunto de serviços de urgência não hospitalares, devendo atender 24 horas por dia, nos sete dias da semana, e contar com equipe multiprofissional; (ii) atenção ambulatorial às pessoas em situação de violência sexual (classificação 007 do serviço especializado 165); e (iii) interrupção de gravidez nos casos previstos em lei (classificação 006 do serviço especializado 165). ${ }^{44}$

Em relação à assistência à pessoa em situação de violência sexual, destaca-se a notificação compulsória, conforme determinam a Portaria GM/MS $n^{0} 1.271$, de 6 de junho de 2014, ${ }^{45}$ a Portaria GM/MS $n^{0} 204$, de 17 de fevereiro de $2016,{ }^{46}$ e o disposto na Lei n ${ }^{\circ} 8.069$, de 13 de julho de 1990 (dispõe sobre o Estatuto da Criança e do Adolescente e dá outras providências), ${ }^{35}$ na Lei $\mathrm{n}^{0} 10.778$, de 24 de novembro de 2003 (estabelece a notificação compulsória, no território nacional, do caso de violência contra a mulher que for atendida em serviços de saúde públicos ou privados) ${ }^{47}$ e na
Lei $\mathrm{n}^{0} 10.741$, de $1^{\circ}$ de outubro de 2003 (dispõe sobre 0 Estatuto do Idoso e dá outras providências). ${ }^{48}$

No caso de violência sexual e tentativas de suicídio, a notificação deve se realizar em até 24 horas na esfera municipal, com o objetivo de garantir a intervenção oportuna nos casos. ${ }^{45,46}$ A notificação imediata é fundamental para a organização dos serviços e para a garantia de acesso às medidas de prevenção dos agravos acima comentados, em tempo oportuno. A notificação dar-se-á a partir do fluxo definido pela vigilância local: o serviço de saúde preenche a ficha específica do Sistema de Informação de Agravos de Notificação (Sinan) e a encaminha à vigilância municipal, a qual segue o fluxo para a vigilância estadual e, posteriormente, à Secretaria de Vigilância em Saúde do Ministério da Saúde. ${ }^{45,46}$

\section{Contribuição dos autores}

Menezes MLB, Araújo MAL, Santos ASD, Gir E e Bermúdez XPD contribuíram igualmente com a concepção, delineamento, redação e revisão crítica do manuscrito. Todos os autores aprovaram a versão final do trabalho e são responsáveis por todos os seus aspectos, incluindo a garantia de sua precisão e integridade.

\section{Agradecimento}

Os autores agradecem a contribuição para a realização deste trabalho aos membros do grupo técnico de especialistas responsáveis pela elaboração do PCDT para Atenção Integral às Pessoas com IST em 2020.

\section{Referências}

1. Brasil. Ministério da Saúde. Portaria $n^{\circ} 42$, de 05 de outubro de 2018. Torna pública a decisão de aprovar o Protocolo Clínico e Diretrizes Terapêuticas para Atenção Integral às Pessoas com Infec ções Sexualmente Transmissíveis (IST), no âmbito do Sistema Único de Saúde - SUS [Internet]. Diário Oficial da União, Brasília (DF), 2018 out 8 [citado 2020 out 26]: Seção I:88. Disponível em: http://bvsms.saude.gov.br/ bvs/saudelegis/sctie/2018/prt0042_08_10_2018.html.

2. World Health Organization - WHO, Etienne GK, Linda LD, James AM, Anthony BZ, Rafael L. World report on violence and health, 2002. Sexual violence (Chapter 6). World report on violence and health

[Internet]. Geneva: WHO; 2002 [cited 2020 0ct 26]. Available from: www.who.int/violence_injury_prevention/violence/global_campaign/en/chap6.pdf.

3. Lutgendorf MA. Intimate partner violence and women's health. Obstet Gynecol [Internet]. 2019 Sep [cited 2020 0ct 26];134(3):470-80. Available from: https://doi.org/10.1097/aog.0000000000003326.

4. Tyson V. Understanding the personal impact of sexual violence and assault. J Women Politics Policy [Internet]. 2019 Mar [cited 2020 0ct 26];40(1):174-83. Available from: https://doi. org/10.1080/1554477X.2019.1565456. 
5. Gomes R, Murta D, Facchini R, Meneghel SN. Gênero, direitos sexuais e suas implicações na saúde. Ciênc Saúde Coletiva [Internet]. 2018 Jun [cited 2020 Sep 14];23(6):1997-2006. Available from: http:// dx.doi.org/10.1590/1413-81232018236.04872018.

6. Fórum Brasileiro de Segurança Pública. Anuário brasileiro de segurança pública 2019 [Internet]. [São Paulo]: Fórum Brasileiro de Segurança Pública; 2019 [citado 2020 jul 23]. 218 p. Disponível em: https:/www.forumseguranca.org.br/wp-content/ uploads/2019/10/Anuario-2019-FINAL_21.10.19.pdf.

7. Ministério da Saúde (BR). Ministério da Justiça (BR). Secretaria de Políticas para as Mulheres. Atenção humanizada às pessoas em situação de violência sexual com registro de informações e coleta de vestígios: norma técnica [Internet]. Brasília: Ministério da Saúde; 2015 [citado 2020 out 26]. Disponível em: https:// bvsms.saude.gov.br/bvs/publicacoes/atencao_humanizada_pessoas_violencia_sexual_norma_tecnica.pdf.

8. Brasil. Presidência da República. Decreto-Lei $n^{0}$ 2.848, de 7 de dezembro de 1940. Código Penal [Internet]. Diário Oficial da União, Rio de Janeiro (RJ), 1940 dez 31 [citado 2020 jun 24]; Seção 1:4. Disponível em: https://www.planalto. gov.br/ccivil_03/decreto-lei/del2848.htm.

9. Ministério da Saúde (BR). Secretaria de Vigilância em Saúde. Violências contra mulheres: análise das notificações realizadas no setor saúde, Brasil, 2011-2018. Bol Epidemiol [Internet]. 2019 out [citado 2020 out 26];50(3). Disponível em: https:// antigo.saude.gov.br/images/pdf/2019/novembro/07/Boletim-epidemiologico-SVS-30.pdf.

10. Sabidó M, Kerr LR, Mota RS, Benzaken AS, Pinho AAP, Guimaraes MDC, et al. Sexual violence against men who have sex with men in Brazil: a respondent-driven sampling survey. AIDS Behav [Internet]. 2015 Sep [cited 2020 Oct 26];19(9):1630-41. Available from: https://doi.org/10.1007/s10461-015-1016-Z.

11. Zalewski M, Drumond P, Prügl E, Setern M. Sexual violence against men in global politics. New York: Routledge; 2018.

12. Drumond $\mathrm{P}$. What about men? Towards a critical interrogation of sexual violence against men in global politics. Int Affairs [Internet]. 2019 Nov [cited 2020 0ct 26];95(6):1271-87. Available from: https://doi.org/10.1093/ia/iiz178.

13. Chynoweth SK, Freccero J, Touquet H. Sexual violence against men and boys in conflict and forced displacement: implications for the health sector. Reprod Health Matters [Internet]. 2017 [cited 2020 0ct 26];25(51):90-4. Available from: https://doi.org/10.1080/09688080.2017.140189.

14. Weare S, Porter J, Evans E. Forced-to-penetrate cases: lived experiences of men. Baseline Research Findings [Internet]. Lancaster: Lancaster University; 2017 [cited 2020 0ct 26]. 15 p. Available from: https://wp.lancs.ac.uk/forced-to-penetrate-cases/files/2016/11/Project-Report-Final.pdf.

15. Bueno S, Pereira C, Neme C. A invisibilidade da violência sexual no Brasil. In: Fórum Brasileiro de Segurança Pública. Anuário brasileiro de segurança pública 2019 [Internet]. [São Paulo]: Fórum Brasileiro de Segurança Pública; 2019 [citado 2020 jul 23]. p. 116-21. Disponível em: https://www.forumseguranca.org.br/wp-content/ uploads/2019/10/Anuario-2019-FINAL_21.10.19.pdf.

16. Blondeel K, Vasconcelos S, García-Moreno C, Stephenson R, Temmerman M, Toskin I. Violence motivated by perception of sexual orientation and gender identity: a systematic review. Bull World Health Organ [Internet]. 2018 Jan [cited 2020 0ct 26];96(1):29-41. Available from: https://doi.org/10.2471/BLT.17.197251.

17. Delziovo CR, Coelho EBS, d'Orsi E, Lindner SR. Violência sexual contra a mulher e 0 atendimento no setor saúde em Santa Catarina - Brasil. Ciênc Saúde Coletiva [Internet]. 2018 [citado 2020 out 26];23(5):1687-96. Disponível em: http://dx.doi. org/10.1590/1413-81232018235.20112016.

18. Ministério da Saúde (BR). Secretaria de Vigilância em Saúde. Departamento de Vigilância, Prevenção e Controle das Infecções Sexualmente Transmissíveis, do HIV/ Aids e das Hepatites Virais. Protocolo clínico e diretrizes terapêuticas para profilaxia pós-exposição de risco (PEP) à infecção pelo HIV, IST e hepatites virais [Internet]. Brasília: Ministério da Saúde; 2018 [citado 2020 out 26]. Disponível em: http://www.aids.gov.br/pt-br/ pub/2015/protocolo-clinico-e-diretrizes-terapeuticas-para-profilaxia-pos-exposicao-pep-de-risco.

19. Mascarenhas MDM, Tomaz GR, Meneses GMS, Rodrigues MTP, Pereira VOM, Corassa RB. Análise das notificações de violência por parceiro íntimo contra mulheres, Brasil, 2011-2017. Rev Bras Epidemiol [Internet]. 2020 Jul [citado 2020 out 26];23(Suppl 01). Disponível em: https:// doi.org/10.1590/1980-549720200007.supl.1. 
20. Casey EA, Masters NT, Querna K, Beadnell B, Hoppe MJ, Morrison DM, et al. Patterns of intimate partner violence and sexual risk behavior among young heterosexually active men. J Sex Res [Internet]. 2016 [cited 2020 0ct 26];53(2):239-50. Available from: https://doi.org/10.1080/00224499.2014.1002125.

21. Bagwell-Gray ME. Women's healing journey from intimate partner violence: establishing positive sexuality. Qual Health Res [Internet]. 2019 May [cited 2020 0ct 26];29(6):779-95. Available from: https://doi.org/10.1177/1049732318804302.

22. Ministério da Saúde (BR). Secretaria de Vigilância em Saúde. Departamento de Doenças de Condições Crônicas e Infecções Sexualmente Transmissíveis. Protocolo clínico e diretrizes terapêuticas para atenção integral às pessoas com infecções sexualmente transmissíveis (IST) [Internet]. Brasília: Ministério da Saúde; 2020 [citado 2020 out 26]. Disponível em: http://www.aids.gov.br/pt-br/ pub/2015/protocolo-clinico-e-diretrizes-terapeuticas-para-atencao-integral-pessoas-com-infeccoes.

23. Ministério da Saúde (BR). Secretaria de Atenção à Saúde. Departamento de Ações Programáticas Estratégicas. Prevenção e tratamento dos agravos resultantes da violência sexual contra mulheres e adolescentes: norma técnica [Internet]. 3. ed. Brasília: Ministério da Saúde; 2012 [citado 2020 out 26]. Disponível em: https://bvsms.saude.gov.br/bvs/publicacoes/prevencao_agravo_violencia_sexual_mulheres_3ed.pdf.

24. O'Byrne P, Orser L, Vandyk A. Immediate PrEP after PEP: results from an observational nurse-led PEP2PrEP study. J Int Assoc Provid AIDS Care [Internet]. 2020 Jan-Dec [cited 2020 0ct 26];19:2325958220939763. Available from: https://doi.org/10.1177/2325958220939763.

25. Ministério da Saúde (BR). Secretaria de Vigilância em Saúde. Departamento de Vigilância, Prevenção e Controle das Infecções Sexualmente Transmissíveis, do HIV/Aids e das Hepatites Virais. Prevenção Combinada do HIV/Bases conceituais para profissionais, trabalhadores(as) e gestores(as) de saúde. Brasília: Ministério da Saúde; 2017 [citado 2020 out 26]. Disponível em: http://www.aids.gov.br/pt-br/pub/2017/ prevencao-combinada-do-hiv-bases-conceituais-para-profissionais-trabalhadoresas-e-gestores.

26. Centers for Disease Control and Prevention - CDC. updated guidelines for antiretroviral postexposure prophylaxis after sexual, injection drug use, or other nonoccupational exposure to
HIV [Internet]. Atlanta: Centers for Disease Control and Prevention; 2016 [cited 2020 Jul 23]. 91 p. Available from: https://www.cdc.gov/HIV/pdf/ programresources/cdc-HIV-npep-guidelines.pdf.

27. European AIDS Clinical Society . Guidelines: version 8.0 [Internet]. London: European AIDS Clinical Society; 2015 [cited 2020 Jul 23]. 94 p. Available from: https:/www.eacsociety.org/ files/guidelines_8_0-english_web.pdf.

28. Hurst SA, Appelgren KE, Kourtis AP. Prevention of mother-to-child transmission of HIV type 1: the role of neonatal and infant prophylaxis. Expert Rev Anti Infect Ther [Internet]. 2015 [cited 2020 Oct 26];13(2):169-81. Available from: https:// doi.org/10.1586/14787210.2015.999667.

29. Bucher MK, Spatz DL. Ten-year systematic review of sexuality and breastfeeding in medicine, psychology, and gender studies. Nurs Womens Health [Internet]. 2019 [cited 2020 0ct 26];23(6):494-507. Available from: https:// doi.org/10.1016/j.nwh.2019.09.006.

30. Chiesa A, Goldson E. Child sexual abuse. Pediatr Rev [Internet]. 2017 [cited 2020 0ct 26];38(3):105-18. Available from: https://doi.org/10.1542/pir.2016-0113.

31. Vrolijk-Bosschaart TF, Brilleslijper-Kater SN, Benninga MA, Lindauer R, Teeuw AH. Clinical practice: recognizing child sexual abuse-what makes it so difficult? Eur J Pediatr [Internet]. 2018 [cited 2020 0ct 26];177(9):1343-50. Available from: https://doi.org/10.1007/s00431-018-3193-Z.

32. Adams JA, Farst KJ, Kellogg ND. Interpretation of medical findings in suspected child sexual abuse: an update for 2018. J Pediatr Adolesc Gynecol [Internet]. 2018 [cited 2020 0ct 26];31(3):225-31. Available from: https://doi.org/10.1016/j.jpag.2017.12.011.

33. Rogstad KE, Wilkinson D, Robinson A. Sexually transmitted infections in children as a marker of child sexual abuse and direction of future research. Curr Opin Infect Dis [Internet]. 2016 [cited 2020 0ct 26];29(1):41-4. Available from: https:// doi.org/10.1097/QC0.0000000000000233.

34. Ministério da Saúde (BR). Secretaria de Vigilância em Saúde. Departamento de Vigilância, Prevenção e Controle das Infecções Sexualmente Transmissíveis, do HIV/Aids e das Hepatites Virais. Protocolo clínico e diretrizes terapêuticas para manejo da infecção pelo HIV em crianças e adolescentes [Internet]. Brasília: Ministério da Saúde; 2018 [citado 2020 out 
26]. Disponível em: http://www.aids.gov.br/pt-br/ pub/2017/protocolo-clinico-e-diretrizes-terapeuticas-para-manejo-da-infeccao-pelo-hiv-em-criancas-e.

35. Brasil. Casa Civil. Lei $n^{\circ} 8.069$, de 13 de julho de 1990. Dispõe sobre o Estatuto da Criança e do Adolescente e dá outras providências [Internet]. Diário Oficial da União, Brasília (DF), 1990 jul 13 [citado 2020 jun 24] Seção 1:8. Disponível em: https:// www.planalto.gov.br/ccivil_03/leis/18069.htm.

36. World Health Organization - WHO. Fact sheet on the safety of levonorgestrel-alone emergency contraceptive pills (LNG ECPs) [Internet]. Geneve: World Health Organization; 2010 [cited 2020 Jul 23]. 3 p. Available from: https://apps.who.int/iris/bitstream/handle/10665/70210/WHO_RHR_HRP_10.06_eng.pdf.

37. Ministério da Saúde (BR). Secretaria de Atenção à Saúde. Departamento de Ações Programáticas Estratégicas. Anticoncepção de emergência: perguntas e respostas para profissionais de saúde [Internet]. Brasília: Ministério da Saúde; 2005 [citado 2020 out 26]. Disponível em: http://bvsms.saude.gov.br/ bvs/publicacoes/caderno3_saude_mulher.pdf.

38. Brasil. Ministério da Saúde. Portaria MS/GM n ${ }^{0}$ 2.561, de 23 de setembro de 2020. Dispõe sobre o procedimento de justificação e autorização da interrupção da gravidez nos casos previstos em lei, no âmbito do Sistema Único de Saúde-SUS [Internet]. Diário Oficial da União, Brasília (DF), 2020 set 23 [citado 2020 out 14]; Seção 1:89. Disponível em: https:/www.in.gov.br/en/web/dou/-/portaria-n-2.561-de-23-de-setembro-de-2020-279185796.

39. Brasil. Presidência da República. Casa Civil. Subchefia para Assuntos Jurídicos. Lei no 12.845, de $1^{\circ}$ de agosto de 2013. Dispõe sobre 0 atendimento obrigatório e integral de pessoas em situação de violência sexual [Internet]. Diário Oficial da União, Brasília (DF), 2013 ago 2 [citado 2020 out 26]. Disponível em: https://presrepublica.jusbrasil. com.br/legislacao/1035667/lei-12845-13.

40. Brasil. Presidência da República. Casa Civil. Subchefia para Assuntos Jurídicos. Decreto 7958, de 13 de março de 13. Estabelece diretrizes para 0 atendimento às vítimas de violência sexual pelos profissionais de segurança pública e da rede de atendimento do Sistema Único de Saúde [Internet]. Diário Oficial da União, Brasília (DF), 2013 mar 14 [citado 2020 out 26]. Disponível em: https://presrepublica.jusbrasil. com.br/legislacao/1034354/decreto-7958-13.
41. Brasil. Ministério da Saúde. Portaria n ${ }^{\circ}$ 485, de $1^{\circ}$ de abril de 2014 . Redefine o funcionamento do Serviço de Atenção às Pessoas em Situação de Violência Sexual no âmbito do Sistema Único de Saúde (SUS) [Internet]. Diário Oficial da União, Brasília (DF), 2014 abr 1 [citado 2020 jul 23];Seção 1:2. Disponível em: http://bvsms.saude.gov.br/bvs/ saudelegis/gm/2014/prt0485_01_04_2014.html.

42. Ministério da Saúde (BR). Secretaria de Atenção à Saúde. Departamento de Atenção Básica. Protocolos da atenção básica: saúde das mulheres [Internet]. Brasília: Ministério da Saúde; 2016 [citado 2020 out 26]. Disponível em: http:// bvsms.saude.gov.br/bvs/publicacoes/protocolos_atencao_basica_saude_mulheres.pdf.

43. Organização Mundial da Saúde - OMS. Relatório mundial sobre a prevenção da violência 2014 [Internet]. São Paulo: Organização Mundial da Saúde; 2020 [citado 2020 jul 23]. 288 p. Disponível em: https://nev.prp.usp.br/wp-content/uploads/2015/11/ 1579-VIP-Main-report-Pt-Br-26-10-2015.pdf.

44. Brasil. Ministério da Saúde. Portaria MS/GM n ${ }^{\circ} 618$, de 18 de julho de 2014. Altera a tabela de serviços especializados do Sistema de Cadastro Nacional de Estabelecimentos de Saúde (SCNES) para o serviço 165 Atenção Integral à Saúde de Pessoas em Situação de Violência Sexual e dispõe sobre regras para seu cadastramento [Internet]. Diário Oficial da União, Brasília (DF), 2014 jul 14 [citado 2020 jul 23]; Seção 1. Disponível em: http://bvsms.saude.gov.br/bvs/ saudelegis/sas/2014/prt0618_18_07_2014.html.

45. Brasil. Ministério da Saúde. Portaria MS/GM n ${ }^{0}$ 1.271, de 6 de junho de 2014. Define a Lista Nacional de Notificação Compulsória de doenças, agravos e eventos de saúde pública nos serviços de saúde públicos e privados em todo o território nacional, nos termos do anexo, e dá outras providências [Internet]. Diário Oficial da União, Brasília (DF), 2014 jun 6 [citado 2020 jul 23];Seção 1. Disponível em: http://bvsms.saude.gov.br/bvs/ saudelegis/gm/2014/prt1271_06_06_2014.html.

46. Brasil. Ministério da Saúde. Gabinete do Ministro. Portaria MS/GM n ${ }^{0}$ 204, de 17 de fevereiro de 2016. Define a Lista Nacional de Notificação Compulsória de doenças, agravos e eventos de saúde pública nos serviços de saúde públicos e privados em todo 0 território nacional, nos termos do anexo, e dá outras providências. [Internet]. Diário Oficial da União, Brasília (DF), 2016 fev 18 [citado 2020 set 17]; Seção 1. 
Disponível em: http://saude.gov.br/images/pdf/2016/ fevereiro/22/Portarias-204-e-205-de-17-02-2016---Lista-Nacional-de-Notifica----o-Compuls--ria-e-Lista-Monitoramento-Unidades-Sentinelas.pdf.

47. Brasil. Presidência da República. Casa Civil. Subchefia para Assuntos Jurídicos. Lei $n^{0} 10.778$, de 24 de novembro de 2003. Estabelece a notificação compulsória, no território nacional, do caso de violência contra a mulher que for atendida em serviços de saúde públicos ou privados. Diário

\begin{abstract}
This article addresses sexual violence, a topic that makes up the Clinical Protocol and Therapeutic Guidelines for Comprehensive Care for People with Sexually Transmitted Infections, published by the Ministry of Health of Brazil. Guidance is given to managers in programmatic and operational management with a focus on the service network for people in situations of sexual violence, recommendations to bealth professionals about prophylactic measures of pregnancy and viral and non-viral sexually transmitted infections, in addition to strategies for surveillance actions. Sexual violence is a broad problem that goes beyond the bealth field and involves conceptual and programmatic challenges for both health professionals, who are at the forefront of care for affected people, and for society, in terms of possible forms of prevention.
\end{abstract}

Keywords: Sexual Offenses; Intimate Partner Violence; Sexually Transmitted Diseases; Clinical Protocols.
Oficial da União, Brasília (DF), 2003 nov 25 [citado 2020 out 26]. Disponível em: http://www. planalto.gov.br/ccivil_03/Leis/2003/L10.778.htm.

48. Brasil. Presidência da República. Casa Civil. Subchefia para Assuntos Jurídicos. Lei ${ }^{0} 10.741$, de $1^{\circ}$ de outubro de 2003. Dispõe sobre o Estatuto do Idoso e dá outras providências [Internet]. Diário Oficial da União, Brasília (DF), 2003 out 3 [citado 2020 out 26]. Disponível em: http://www.planalto. gov.br/ccivil_03/LEIS/2003/L10.741.htm.

\section{Resumen}

Este artículo aborda la violencia sexual, tema que integra el Protocolo Clínico y Lineamientos Terapéuticos para la Atención Integral a Personas con Infecciones de Transmisión Sexual, publicado por el Ministerio de Salud de Brasil. Se brinda orientación a los gerentes en la gestión programática y operativa con enfoque en la red de servicios para personas en situación de violencia sexual, recomendaciones a los profesionales de la salud sobre medidas profilácticas del embarazo e infecciones de transmisión sexual virales y no virales, además de estrategias para acciones de vigilancia. La violencia sexual es un problema amplio que va más allá del campo de la salud e implica desafios conceptuales y programáticos tanto para los profesionales de la salud, que están a la vanguardia de la atención a las personas afectadas, como para la sociedad, en cuanto a posibles formas de prevención.

Palabras clave: Delitos Sexuales; Violencia de Pareja; Infecciones de Transmisión Sexual; Enfermedades de Transmisión Sexual; Protocolos Clínicos. 


\section{Errata}

No artigo "Protocolo Brasileiro para Infecções Sexualmente Transmissíveis 2020: violência sexual", doi: 10.1590/S1679-4974202100018.esp1, Figura 1 - Esquema profilático para infecções sexualmente transmissíveis não virais em situações de violência sexual, publicado no periódico Epidemiologia e Serviços de Saúde, v. 30(Esp.1):1-11, na página 4:

Onde se lia:

\begin{tabular}{|l|l|l|l|}
\hline \multirow{2}{*}{$\begin{array}{l}\text { Infecção sexualmente } \\
\text { transmissível }\end{array}$} & Medicamento & \multicolumn{2}{|c|}{ Posologia } \\
\cline { 3 - 4 } Sífilis & Adultos e adolescentes com mais & $\begin{array}{l}\text { Crianças e adolescentes com } \\
\text { menos de 45kg }\end{array}$ \\
\hline Gonorreia & Benzilpenicilina benzatina & $\begin{array}{l}2,4 \text { milhões de unidades internacionais } \\
\text { (UI), intramuscular (IM) em dose única } \\
\text { (dividida em duas injeções) }\end{array}$ & $\begin{array}{l}50.000 \mathrm{UI} / \mathrm{kg}, \text { IM, dose única (dose } \\
\text { máxima total: 2,4 milhões UI) }\end{array}$ \\
\hline Infecção por clamídia & Ceftriaxona + azitromicina & $\begin{array}{l}\text { Ceftriaxona 500mg, 1 ampola, IM, } \\
\text { dose única }\end{array}$ & $+/+++$ \\
\hline Tricomoníase & Azitromicina & $\begin{array}{l}500 \mathrm{mg}, 2 \text { comprimidos, V0, dose única } \\
\text { (dose total: } 1 \mathrm{~g})\end{array}$ & $\begin{array}{l}20 \mathrm{mg} / \mathrm{kg} \text { de peso, V0, dose única (dose } \\
\text { máxima total: 1g) }\end{array}$ \\
\hline
\end{tabular}

Leia-se:

\begin{tabular}{|c|c|c|c|}
\hline \multirow{2}{*}{$\begin{array}{l}\text { Infecção sexualmente } \\
\text { transmissível }\end{array}$} & \multirow[b]{2}{*}{ Medicamento } & \multicolumn{2}{|c|}{ Posologia } \\
\hline & & $\begin{array}{l}\text { Adultos e adolescentes com mais de } \\
45 \mathrm{~kg} \text {, inclusive gestantes }\end{array}$ & $\begin{array}{l}\text { Crianças e adolescentes com } \\
\text { menos de } 45 \mathrm{~kg}\end{array}$ \\
\hline Sífilis & Benzilpenicilina benzatina & $\begin{array}{l}\text { 2,4 milhões de unidades internacionais (UI), } \\
\text { intramuscular (IM) em dose única (dividida } \\
\text { em duas injeções) }\end{array}$ & $\begin{array}{l}50.000 \text { UI/kg, IM, dose única (dose } \\
\text { máxima total: } 2,4 \text { milhões UI) }\end{array}$ \\
\hline Gonorreia & Ceftriaxona + azitromicina & $\begin{array}{l}\text { Ceftriaxona 500mg, } 1 \text { ampola, IM, } \\
\text { dose única mais azitromicina 500mg, } 2 \\
\text { comprimidos, via oral (V0), dose única } \\
\text { (dose total: } 1 \mathrm{~g} \text { ) }\end{array}$ & $\begin{array}{l}\text { Ceftriaxona } 125 \mathrm{mg}, \mathrm{IM} \text {, dose única mais } \\
\text { azitromicina } 20 \mathrm{mg} / \mathrm{kg} \text { de peso, V0, dose } \\
\text { única (dose máxima total: } 1 \mathrm{~g} \text { ) }\end{array}$ \\
\hline Infecção por clamídia & Azitromicina & $\begin{array}{l}\text { 500mg, } 2 \text { comprimidos, V0, dose única } \\
\text { (dose total: } 1 \mathrm{~g} \text { ) }\end{array}$ & $\begin{array}{l}20 \mathrm{mg} / \mathrm{kg} \text { de peso, } \mathrm{V} 0 \text {, dose única (dose } \\
\text { máxima total: } 1 \mathrm{~g} \text { ) }\end{array}$ \\
\hline Tricomoníase & Metronidazo ${ }^{\mathrm{a}, \mathrm{b}}$ & $\begin{array}{l}\text { 500mg, } 4 \text { comprimidos V0, dose única } \\
\text { (dose total: } 2 \mathrm{~g} \text { ) }\end{array}$ & $\begin{array}{l}15 \mathrm{mg} / \mathrm{kg} / \text { dia, divididos em } 3 \text { doses/ } \\
\text { dia, por } 7 \text { dias (dose diária máxima: } 2 \mathrm{~g} \text { ) }\end{array}$ \\
\hline
\end{tabular}

